\title{
Hyperplasia and Metaplasia
}

National Cancer Institute

\section{Source}

National Cancer Institute. Hyperplasia and Metaplasia. NCI Thesaurus. Code C120890.

A finding of both hyperplastic and metaplastic changes in a pathologic process. 